\title{
A 10-day mild treadmill exercise performed before an epileptic seizure alleviates oxidative injury in the skeletal muscle and brain tissues of the rats
}

\author{
Sevil ARABACI-TAMER ${ }^{1}$ (D), Ozlem Tugce CILINGIR-KAYA² (D), Meral YUKSEL ${ }^{3}$ (D), Alper YILDIRIM ${ }^{4}$ (D), Berrak C. YEGEN ${ }^{4}$ \\ ${ }^{1}$ Department of Physiology, School of Medicine, Sakarya University, Sakarya, Turkey. \\ ${ }^{2}$ Department of Histology and Embryology, School of Medicine, Marmara University, Istanbul, Turkey. \\ ${ }^{3}$ Vocational School of Health Sciences, Marmara University, Istanbul, Turkey. \\ ${ }^{4}$ Department of Physiology, School of Medicine, Marmara University, Istanbul, Turkey.
}

Corresponding Author: Alper YILDIRIM

E-mail: kemalper@yahoo.com

Submitted: 08.08.2021 Accepted: 05.11.2021

\begin{abstract}
Objective: Epileptic seizures may cause skeletal muscle injury and memory dysfunctions. The present study was aimed to investigate the possible protective effects of exercising prior to seizure on seizure-induced oxidative injury in the skeletal muscle and brain.

Materials and Methods: Sprague-Dawley male rats were assigned as non-exercise $(n=16)$ and exercise groups $(n=16)$. Following a 3-day exercise training, exercise protocol (30 min) was performed on a treadmill for 10 days, while control rats had no exercise. On the 11th day, the epileptic seizure was induced by a single intraperitoneal injection of pentylenetetrazol (PTZ) (45 mg/kg), while the control groups were injected with saline. Passive-avoidance test was initially performed before PTZ/saline injection and repeated $72 \mathrm{~h}$ later for the assessment of memory function. Brain and gastrocnemius muscles were taken for histological assessments and to determine the levels of malondialdehyde (MDA) and glutathione (GSH), myeloperoxidase (MPO) activity and luminal - and lucigenin - enhanced chemiluminescence levels.

Results: Exercise training alone increased the formation of reactive oxygen species and elevated the antioxidant GSH capacity of the muscle tissue in the control rats, but these effects were not observed in the muscles of the exercised rats induced with a PTZ-seizure. On the other hand, short-term exercise alone had no effect on the basal oxidative parameters of the brain tissues. Prior exercise did not alter the average seizure scores or memory performances when compared to non-exercised groups, but suppressed the PTZinduced elevations in MDA and chemiluminescence levels as well as MPO activity in the brain.

Conclusion: A 10-day mild treadmill exercise reduced the oxidative brain damage due to a single seizure-induced excitotoxicity and exerted a preconditioning effect on the skeletal muscles exposed to tonic-clonic contractions.

Keywords: Epileptic seizure, Exercise, Oxidative damage, Memory dysfunction, Skeletal muscle
\end{abstract}

\section{INTRODUCTION}

Status epilepticus (SE) is a common life-threatening neurological emergency characterized by a prolonged seizure, which results in excitotoxicity-induced cerebral injury [1]. Neuronal hyperexcitability during SE causes oxidative stress, presenting with increased generation of reactive oxygen species (ROS), lipid peroxidation [2], and consequently with neuronal degeneration that includes the hippocampal areas, resulting in cognitive dysfunction and deficits in memory and learning [36]. Apart from the brain tissue, generalized excessive muscle contractions in SE affect all the organ systems, particularly the over-working skeletal muscles [7]. Moreover, increased load on the skeleton due to exaggerated muscle contractions during seizures may even cause fractures, skeletal muscle damage, and rhabdomyolysis [8-11].

In contrast to limited information confirming the prevalence of physical exercise as a seizure-triggering factor and the lack of any mechanistical explanations regarding how exercise could induce seizures [12], exercise was commonly suggested to have several beneficial effects on the outcomes of epileptic seizures in both humans and animals. Although the available studies on the effects of physical activity on seizure frequency are heterogeneous, many human studies have generally reported

How to cite this article: Arabaci-Tamer S, Cilingir-Kaya O T, Yuksel M, Yildirim A, Yegen B C. A 10-day mild treadmill exercise performed before an epileptic seizure alleviates oxidative injury in the skeletal muscle and brain tissues of the rats. Marmara Med J 2022; 35(1):1-9. doi: 10.5472/marumj.1056192 
that regular physical exercise can reduce the seizure frequency and improve cardiovascular and psychological health of epileptic patients [13]. Moreover, exercise can exert beneficial actions such as reductions in seizure susceptibility, anxiety and depression, and consequently leading to improvement of life quality of individuals with epilepsy, which suggests that exercise can be a potential candidate for the non-pharmacological treatment of epilepsy $[13,14]$. Accordingly, animal studies have shown that exercise performed before or after the induction of seizure has positive effects on SE. In the early stages of life, longterm memory deficit caused by SE and the occurrence frequency of seizures after SE were reported to be reduced in exercised rodents $[15,16]$. Various exercise regimens were reported to increase latency to SE development, decrease susceptibility to subsequently induced seizures and ameliorate the course of experimentally induced SE $[17,18]$.

In rats, both a preconditioning exercise performed before the induction of ischemic brain injury [19] and an exercise performed for neurorehabilitation during post-ischemic recovery [20] were shown to reduce oxidative brain damage and associated cognitive dysfunctions. Similarly, exercise training was proven to stimulate several cellular adaptive events in the skeletal muscle to resist against any injurious insults to the muscle fibers [21]. However, the putative protective role of exercise on the seizure-injured neuronal and muscular tissues was not elucidated before. Thus, our study was aimed to evaluate the possible protective effects of exercising prior to seizure on the seizure-induced oxidative injury of the brain and skeletal muscle.

\section{MATERIALS and METHODS}

\section{Animals}

Sprague-Dawley male rats (240-260 gr) used in the study were supplied by the Marmara University Animal Center (DEHAMER). The rats had free access to a standard rat chow and water, and were kept under laboratory conditions with controlled humidity, temperature $\left(22 \pm 0.5^{\circ} \mathrm{C}\right)$ and light/dark $(12 / 12 \mathrm{~h})$ cycles. All experimental protocols, guided by the National Institute of Health guidelines for the Care and the Use of Laboratory Animals, were approved by the Marmara University Animal Care and Use Committee (approval code: 77.2017.mar; date: 6.11.2017).

\section{Experimental design and exercise}

The rats were randomly assigned to either non-exercise $(n=16)$ or exercise groups $(n=16)$ (Fig. 1$)$. In the exercise groups, initially a 3-day adaptation period for $10 \mathrm{~min} /$ day was allowed for the rats to get accustomed to the exercise procedure by placing them on a rodent treadmill (TME 9805, Commat Instruments, Ankara, Turkey). After the acclimatization period, the exercising rats ran on a treadmill at $0^{\circ}$ of inclination initially at a speed of $10 \mathrm{~m} /$ min (first 3 days) and then at $11.6 \mathrm{~m} / \mathrm{min}$ (following 7 days) speed for $30 \mathrm{~min} /$ day for 10 days [22], while the sedentary rats had no exercise training. Following the 10 -day exercise training period, an epileptic seizure was induced on the $11^{\text {th }}$ day by the intraperitoneal injection of pentylenetetrazol (PTZ), while the control groups were injected with saline. Since the aim was to evaluate only the preconditioning effects of exercise on seizureinduced oxidative damage, exercise was discontinued during the 3 days following saline or PTZ injection. Passive avoidance test (PAT) was performed on two occasions, immediately before and at the $72^{\text {th }} \mathrm{h}$ of PTZ-seizure, to assess the memory function of the rats. The rats were decapitated after the second PAT performance. Brain and gastrocnemius muscle tissues were collected for histological assessments and to determine the levels of malondialdehyde (MDA) and glutathione (GSH), myeloperoxidase (MPO) activity and chemiluminescence levels of luminol and lucigenin as indicators of reactive oxygen species (ROS) generation.

\section{Seizure induction and assessment}

The rat was placed in a Plexiglas observation box $(38 \times 30 \times$ $25 \mathrm{~cm})$ and a single dose of PTZ (45 mg/kg) was injected intraperitoneally to induce an epileptic seizure [23]. Epileptic seizures were recorded by a video camera for 30 minutes. The intensity of the recorded seizures was evaluated using Racine's scoring system (0-5), where 0, no behavioral changes; 1 , focal facial movements with twitching of ears and whiskers; 2, myoclonic jerks but no rearing up; 3, myoclonic jerks with rearing up; 4, clonic convulsions along with posture loss; 5, generalized tonic-clonic seizures [24].

\section{Evaluation of memory function}

In order to evaluate the effects of exercise on seizure-induced memory dysfunction, PAT was performed using an electronic two-compartment box (Northel Passive Avoidance System, Istanbul, Turkey) composed of an illuminated compartment, a dark compartment and a guillotine gate separating these compartments. On the $11^{\text {th }}$ day of the experimental procedure, all rats underwent the first PAT before PTZ or saline injection (Fig. 1), which was accepted as the acquisition trial of the test [25]. Then, the recall phase of PAT was performed at the $72^{\text {nd }}$ hour of seizure induction just before euthanasia. In the acquisition trial, the rats were placed individually in the illuminated compartment of the box, and due to their inborn tendencies for preference of dark areas, they moved from the illuminated compartment into the dark side and a guillotine door between two chambers was closed. By the electric grid floor of the dark compartment, a mild inescapable electrical foot shock (0.3-0.6 mA) was applied for $5 \mathrm{~s}$, and the rat was then taken out from the box, where it has learned the negative outcomes of entering the dark chamber. Then, $72 \mathrm{~h}$ after this acquisition trial, the rat was put again into the illuminated compartment and the latency to pass to the dark compartment was recorded to assess the memory performance. If the rats have avoided entering the dark chamber with a latency over $300 \mathrm{~s}$ (cut-off point), it was considered as a normal memory performance, while a shorter latency to enter the dark chamber was regarded as poor memory collection [26]. During each of the PAT applications, both compartments were cleaned with $70 \%$ alcohol before the next animal was placed in the box. 


\section{Measurement of myeloperoxidase activity, malondialdehyde and glutathione levels in the skeletal muscle and brain}

Biochemical measurement of MPO activity in a tissue reflects the magnitude of neutrophil infiltration and correlates positively with the histochemically recorded neutrophil infiltration to that tissue [27]. Based on $\mathrm{H} 2 \mathrm{O} 2$-dependent oxidation of o-dianisidine. $2 \mathrm{HCl}, \mathrm{MPO}$ activity in both the skeletal and brain tissues was determined using a spectrophotometer at $460 \mathrm{~nm}$, and the MPO activity was expressed as units per gram tissue [28]. For the determination of MDA and GSH levels, tissue samples were homogenized in trichloroacetic acid solution (10\%) and centrifuged for $15 \mathrm{~min}$ at $3000 \mathrm{~g}$ and $4^{\circ} \mathrm{C}$. The supernatants were mixed with thiobarbituric acid to determine the MDA levels (U/g tissue) as the by-products of lipid peroxidation [29]. GSH levels (nmol/g tissue) in the muscle and brain tissues were determined using a modified Ellman procedure. The absorbance was measured at $412 \mathrm{~nm}$ and the amount of GSH was given as $\mu \mathrm{mol}$ (g/tissue) [30].

\section{Chemiluminescence assays}

Chemiluminescence (CL) assay is a frequently used noninvasive method for the direct assessment of the levels of ROS using a luminometer (Junior LB 9509, EG\&G, Berthold, Germany) at room temperature. Lucigenin-(bis-N methylacridinium nitrate) probe specifically detects superoxide radicals, while luminol5-amino-2,3-dihydro 1,4 phthalazinedione) is used commonly for the detection of $\mathrm{OH}, \mathrm{H} 2 \mathrm{O} 2$ and $\mathrm{HOCl}$ radicals [31]. Using these probes, ROS generation was assessed in both the muscle and brain tissues and CL levels were calculated by linear approximation. ROS generation in terms of CL was expressed as the area under the curve (AUC) of relative light unit per mg tissue [32].

\section{Histopathological examination}

Gastrocnemius muscle samples obtained from the experimental groups were fixed in $4 \%$ paraformaldehyde, processed for routine histological procedures and embedded in paraffin (Leica TP1020 and EG1150H+C). Paraffin sections of $5 \mu \mathrm{m}-$ thick were cut by a rotary microtome (Leica RM2125RT) and stained with hematoxylin and eosin (H\&E) for the light microscopic examinations. The histological features of the muscle sections were evaluated regarding the organization of fibers and the presence of inflammatory cell infiltration and congestion. Myofibers presenting with typical peripheral nuclei were observed in the healthy fibers, whereas centralized nuclei were regarded as a sign of the repairing process of damaged myofibers [33-35].

Following their fixation in $4 \%$ paraformaldehyde, brain tissues were incubated in $20 \%$ sucrose solution at $+4^{\circ} \mathrm{C}$ and were then kept at $-20^{\circ} \mathrm{C}$ until they were cut by a cryostat (Leica CM1950, Germany). Five $\mu \mathrm{m}$-thick frozen sections taken from the hippocampus and cortex were stained with cresyl violet (Nissl staining) for light microscopic examinations. Microscopic appearances of the neurons and the intensity of dark neurons were examined to assess the extent of neuronal injury [36-38].

\section{Statistical Analysis}

Statistical analyses were performed using GraphPad Prism 8.0.2 program (GraphPad Software, San Diego, CA, USA). All data are presented as means \pm SEM. Analysis of the biochemical data was performed by using one-way ANOVA followed by posthoc Tukey test. Values of $\mathrm{p}<0.05$ was considered as statistically significant.

\section{RESULTS}

Following PTZ injection, except for a single rat in the exercised PTZ group that had no seizures at all, the rest of the rats in the non-exercised and exercised groups demonstrated either twitches or tonic-clonic contractions. The averages of the stage scores reached in the exercised and non-exercised groups were similar (Table I). Average seizure scores, the numbers of rats recorded at stages 4 and 5 , and the total duration of contractions during stages 4 and 5 were similar in the non-exercised and exercised rats, showing no difference due to the 10-day exercise performed before an epileptic seizure.

On the acquisition trial of the PAT, no significant differences were observed among the initial latency periods of rats (data not shown). At the recall phase of the PAT, none of the rats in the control groups, either sedentary or exercised, have entered the dark chamber within the cut-off period $(300 \pm 0)$. However, three rats in the sedentary-PTZ rats have entered the dark chamber, while all the rats in the exercise-PTZ group avoided entering into the dark compartment, but no statistically significant difference was observed between the latency periods of the non-exercised or exercised PTZ groups (Table I).

Table I. Seizure scores and passive avoidance test (PAT) results of the rats injected with PTZ

\begin{tabular}{|l|c|c|c|c|}
\hline & \multicolumn{3}{|c|}{ Seizures } & PAT \\
\hline $\begin{array}{c}\text { Average of } \\
\text { stage scores } \\
(1-5)\end{array}$ & $\begin{array}{c}\text { Number of } \\
\text { rats at stage } \\
4-5 \\
(\mathrm{n} ; \%)\end{array}$ & $\begin{array}{c}\text { Total time } \\
\text { spent at stage } \\
4-5 \\
(\mathrm{sec})\end{array}$ & $\begin{array}{c}\text { Retention } \\
\text { latency } \\
(\mathrm{sec})\end{array}$ \\
\hline No exercise & $3.88 \pm 0.58$ & $5 / 8 ; 62.5 \%$ & $224.4 \pm 91.3$ & $232.5 \pm 43.5$ \\
\hline Exercised & $3.50 \pm 0.76$ & $5 / 8 ; 62.5 \%$ & $231.6 \pm 107.2$ & $300 \pm 0$ \\
\hline
\end{tabular}

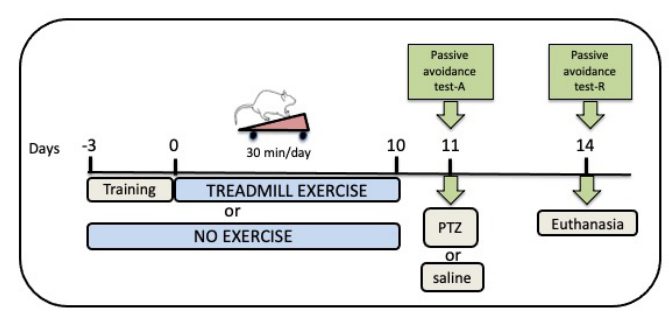

Figure 1. Schematic explanation of the experimental procedures. A: acquisition phase, $R$ : recollection phase of the passive avoidance test. 
Exercise alone had no effect on the oxidative parameters measured in the brain tissues of control rats (Figure 2). However, induction of a PTZ-seizure resulted in the elevation of brain MPO activity, MDA levels, luminol and lucigenin CL levels in the non-exercised rats $(\mathrm{p}<0.01-0.001)$, while a 10 -day exercise performed prior to seizure induction suppressed these elevations significantly $(\mathrm{p}<0.01)$, demonstrating the inhibitory effect of exercise on oxidative brain damage and associated neutrophil infiltration caused by PTZ-seizure. On the other hand, antioxidant glutathione levels in the brain tissues were not altered by neither exercise nor seizure induction.
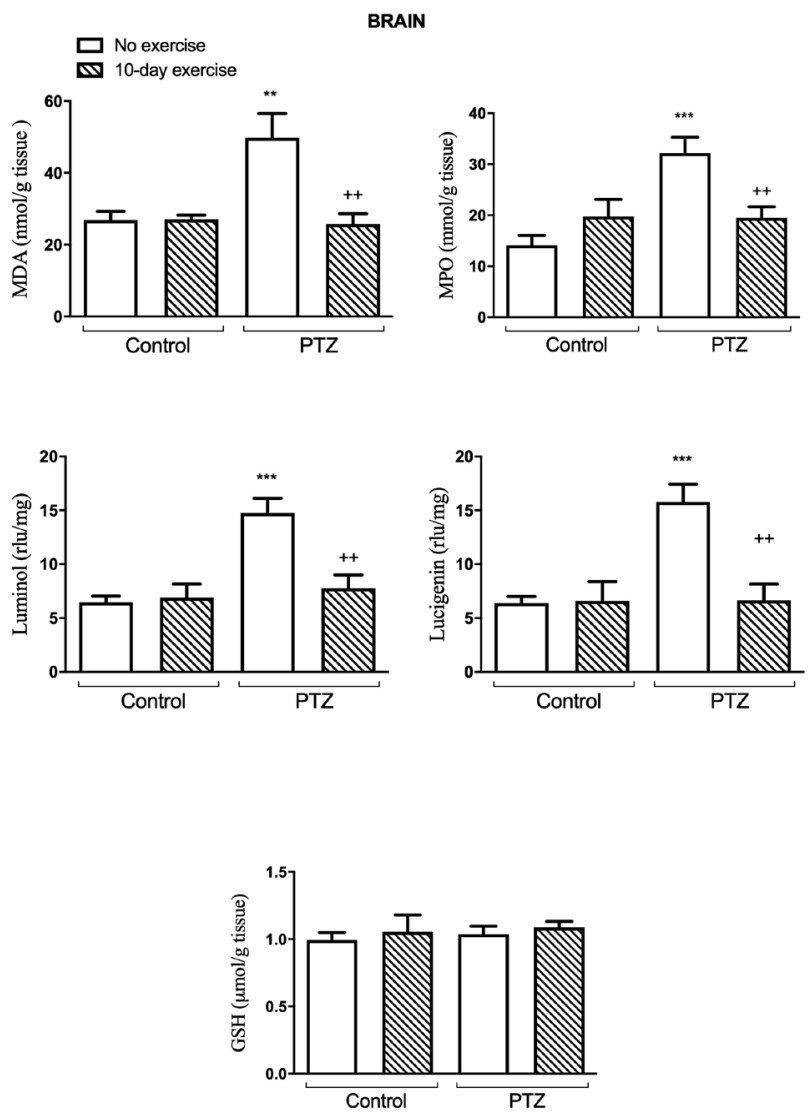

Figure 2. Malondialdehyde (MDA), myeloperoxidase (MPO) activity, glutathione (GSH) content and luminol or lucigenin-enhanced chemiluminescence levels in the brain tissues of the control and PTZseizure groups that have not exercised or had a 10-day treadmill exercise. ${ }^{* *} p<0.01,{ }^{* *} p<0.001$, compared to non-exercised control group; $++p<0.01$ compared to non-exercised PTZ group.

Similar to that observed in the brain tissue, MDA levels and MPO activity in the gastrocnemius muscle of the control rats were not altered by exercise training (Figure 3). However, exercising for 10 days elevated the generation of ROS in the gastrocnemius muscle, as assessed by elevated levels of luminol - $(\mathrm{p}<0.01)$ and lucigenin- $(\mathrm{p}<0.05)$ enhanced CL, which was accompanied by an increase in the antioxidant GSH content $(\mathrm{p}<0.05)$. Although PTZ-seizure in the non-exercised rats did not increase muscle tissue MDA level, it resulted in increased levels of luminol-enhanced CL (indicative of $\mathrm{OH}-, \mathrm{H} 2 \mathrm{O} 2$, $\mathrm{HOCl}$ radicals $)(\mathrm{p}<0.01)$ with a concomitant elevation in the MPO activity $(\mathrm{p}<0.001)$. On the other hand, having exercised prior to PTZ-seizure depressed MPO activity ( $\mathrm{p}<0.01$ ), showing the inhibitory effect of prior exercise on neutrophil infiltration to the muscles that have exhibited tonic-clonic contractions. Despite that exercise in the control rats had a stimulatory impact on antioxidant capacity of the skeletal muscle, PTZ-induced muscle contractions have not changed muscle GSH content in either non-exercised or exercised rats as compared to that of the control rats.
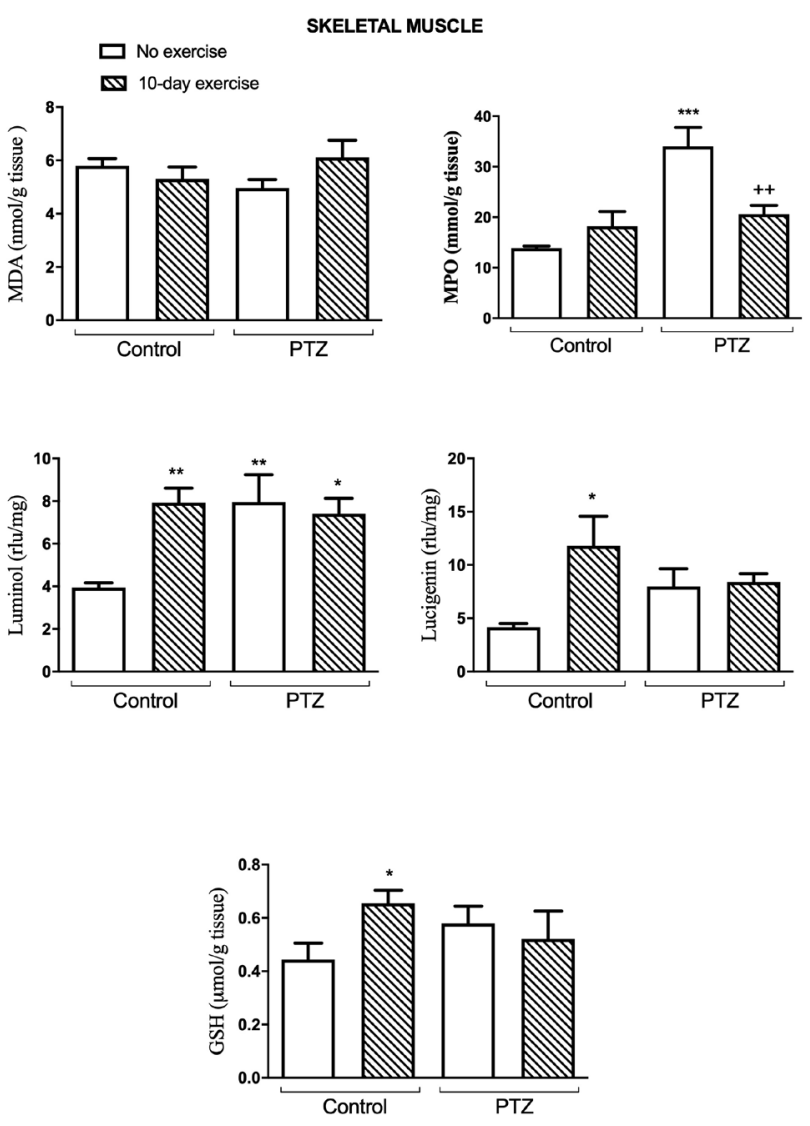

Figure 3. Malondialdehyde (MDA), myeloperoxidase (MPO) activity, glutathione (GSH) content and luminol or lucigenin-enhanced chemiluminescence levels in the gastrocnemius muscle tissues of the control and PTZ-seizure groups that have not exercised or had a 10-day treadmill exercise. ${ }^{*} p<0.05,{ }^{* *} p<0.01,{ }^{* *} p<0.001$, compared to non-exercised control groups; $++p<0.01$ compared to non-exercised PTZ group.

Examination of the H\&E-stained sections of gastrocnemius muscles showed that most of the myofibrils exhibited normal regular morphology with peripherally located nuclei in both the non-exercised and exercised control groups (Figure 4), except for some muscle fibers of the exercised control group presenting with a few abnormally located nuclei, indicating the recovery of the injured muscle tissue [39]. On the other hand, 
in the non-exercised group induced with PTZ, myofibrils with abnormal organization, severe congestion and inflammatory cell infiltration were observed, whereas mild congestion and more regular myofibrils were evident in the exercised PTZ group.

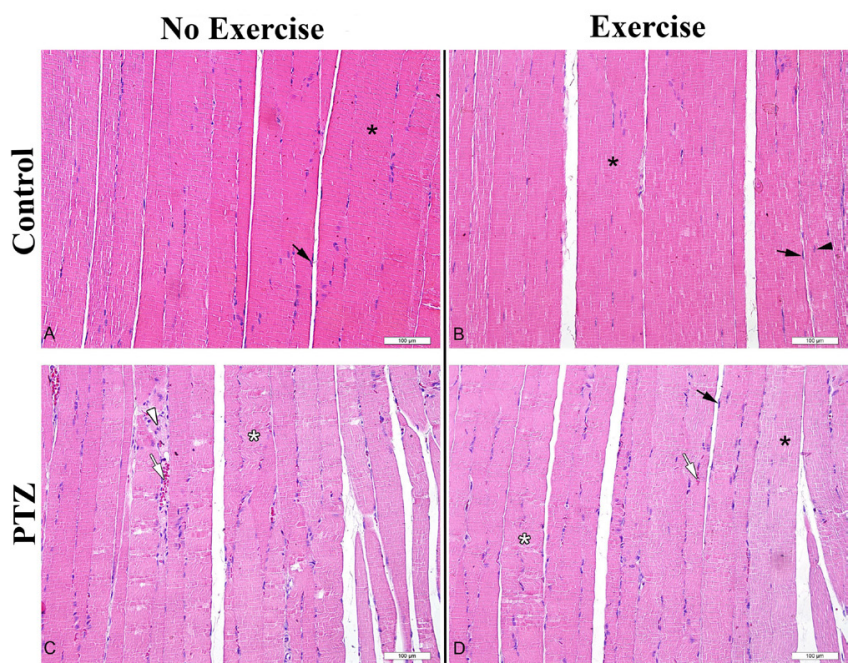

Figure 4. Representative micrographs of the muscle tissues of the experimental groups. Arrow: peripherally located nucleus. Asterisk $\left.{ }^{*}\right)$ : muscle fiber with regular organization. Arrowhead: centrally located nucleus. White asterisk: muscle fiber with an abnormal organization. White arrow: vascular congestion. White arrowhead: inflammatory cell infiltration in the connective tissue. Hematoxylin and eosin staining.

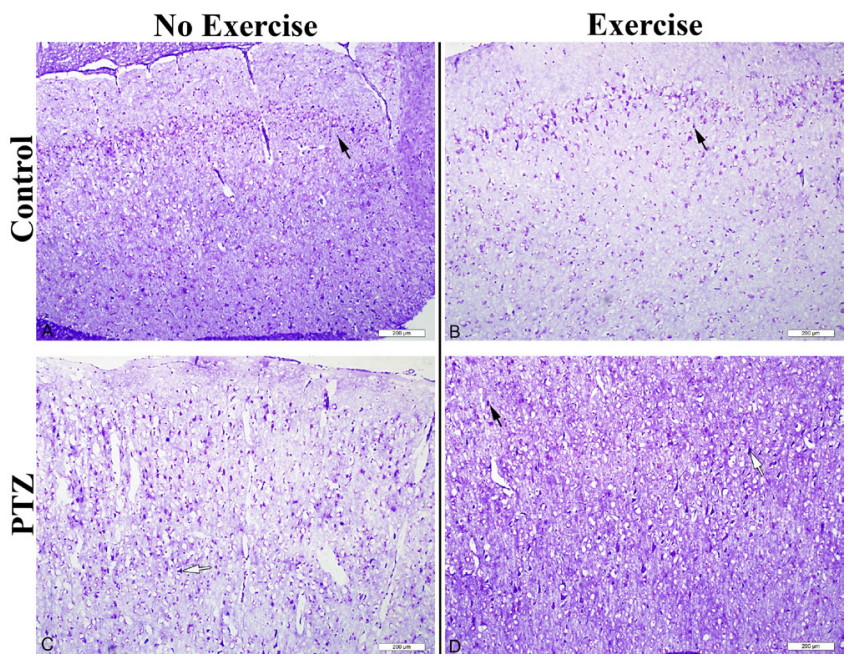

Figure 5. Representative micrographs of cortical regions of the experimental groups. Arrow: regular-shaped neurons. White arrow: Dark neurons with shrunken cell bodies. Cresyl Violet staining.

The evaluation of cresyl violet-stained brain sections of the exercised and non-exercised groups revealed that the neurons in both hippocampus and cortex were round or oval in shape, and consisted of blue nuclei with clearly visible borders, while no remarkable differences were observed between the morphological features of the brains of the exercised or nonexercised control rats (Figures 5 and 6). In the non-exercised group induced with PTZ-seizure, neuronal loss was evident in the dentate gyrus and cornu ammonis regions of the hippocampus, while the strongly stained and shrunken dark neurons were increased in number. In both the hippocampus and cortex, the number of pyramidal neurons and granular cells with indistinguishable borders of the nuclei and the cytoplasm were increased. On the other hand, neuronal morphology in the exercised PTZ group was similar to that observed in the brains of the control rats.

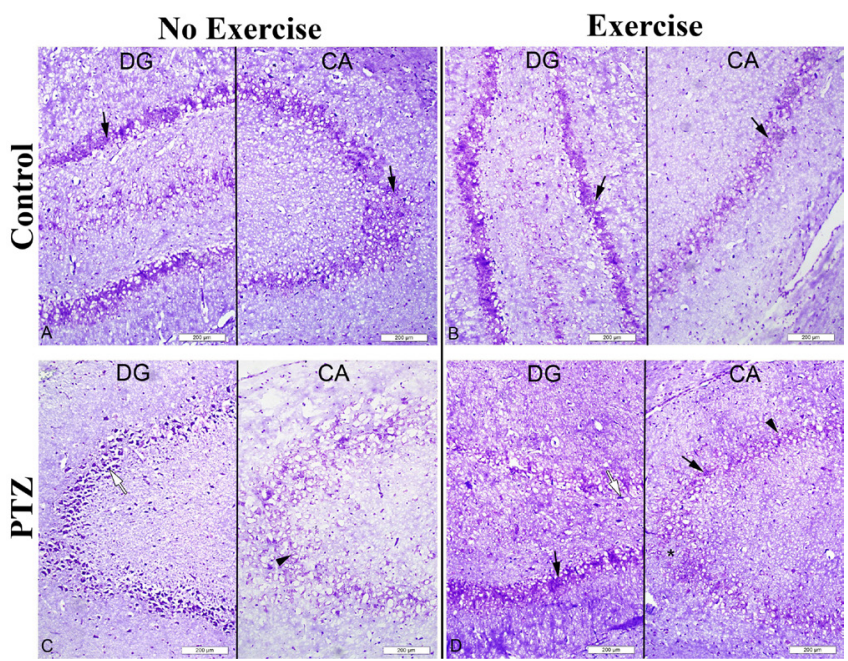

Figure 6. Representative micrographs of the hippocampal regions of the experimental groups. Arrow: round - or oval-shaped neurons. DG: Dentate gyrus; CA: Cornus Ammonis. Arrowhead: degenerated neurons with unclear nuclei and cytoplasms. White arrow: dark neurons with shrunken cell bodies. Asterisk $\left(^{\star}\right)$ : neuron loss. Cresyl Violet staining.

\section{DISCUSSION}

The findings demonstrated that the frequency of tonic-clonic contractions, average seizure scores and memory performances were similar in the non-exercised and exercised groups induced with a PTZ-seizure. Although, exercise training alone increased the formation of ROS and elevated the antioxidant GSH capacity of the muscle tissue in the control rats, these effects were not observed in the muscles of the exercised rats induced with a PTZ-seizure, suggesting that the upregulated GSH provided by prior exercise is depleted to overcome the oxidative injury of the muscle due to its exaggerated activity during seizure. Thereby, exercise prior to seizure was able to suppress PTZinduced elevation in neutrophil infiltration, demonstrating the preconditioning effect of exercise in alleviating seizure-induced oxidative injury of the overworked muscles. In contrast to the skeletal muscle, short-term exercise alone had no effect on the basal oxidative parameters of the brain tissues. However, a 10day mild exercise performed prior to PTZ-seizure was able to reverse the increased lipid peroxidation and ROS generation as well as neutrophil infiltration to the inflamed brain tissue, 
reducing the oxidative brain damage due a single seizureinduced excitotoxicity.

Several neuroprotective and anti-epileptogenic drugs have been examined for seizure prevention and treatment [23, 40]. Among these treatments, physical exercise has been investigated as an effective non-pharmacological complementary therapy for its potency in preventing the occurrence of seizures and in improving the comorbidities of epilepsy [41]. Accordingly, moderate exercise in rats was shown to decrease the spike amplitude and frequency of penicillin-induced seizures [42]. On the other hand, several clinical or experimental studies have suggested that exercise does not significantly affect the nature of seizures. Arida et al., have reported that an acute running exercise for $40 \mathrm{~min}$ in rats did not affect the number of stimulations required to initiate the first generalized seizure [43]. Similarly, it was reported that exercise performed by the epileptic patients has not affected the frequency of their seizures, but had a positive impact on their behavioral states [44]. Furthermore, physical training has been shown to enhance learning and memory in people with epilepsy [45, 46], which was also verified in several animal models [15, 47], suggesting that exercise, owing to its beneficial effects on the cognition and psychology of patients with epilepsy, could be recommended as a life-style change. However, our present results have indicated that a 10-day mild treadmill exercise performed prior to a single seizure did not alter the severity of that seizure and had no impact on the memory function, which was not altered by that single seizure. This discrepancy with the previous studies may be related with the duration and intensity of exercise, as well as the seizure model. On the other hand, our microscopic results revealed that the PTZ-induced degeneration observed in the hippocampal and cortical neurons was abolished in the brains of the exercised rats, indicating that the injury in the microscopical level has not reached to any alteration in the memory function, but along with the addition of other injurious seizures long-term effects of these neurodegenerative changes could be expected to affect the memory function. Since the repetitive epileptic seizures would disturb memory function, regular exercise for longer periods could have a better chance to provide a significant improvement in cognitive functions.

Despite that the performed exercise prior to a PTZ-seizure had no impact on the severity of the seizure, our results have demonstrated that exercise prevented oxidative injury of the brain, as assessed by the suppression of ROS generation, lipid peroxidation and neutrophil infiltration. Overproduction of free radicals due to neuronal hyperexcitability plays a central role in the pathogenesis of epilepsy. Increased production of ROS along with attenuated antioxidant defense makes the brain highly vulnerable to oxidative damage [48]. Since, oxidative damage can alter neuronal functions, the extent of the oxidative stress plays the major role in the pathogenesis and complications of seizure-induced cognitive dysfunctions, including learning and memory disorders [49-51]. We and the others have previously reported that epileptic seizure induced by a single dose of PTZ enhanced lipid peroxidation and neutrophil recruitment, exaggerated ROS generation, while endogenous GSH was depleted in the brain $[23,52,53]$. In the present study, we demonstrated that excess ROS generation in conjunction with increased lipid peroxidation and neutrophil infiltration were reversed by prior exercising demonstrating the neuroprotective effect of exercise, while the antioxidant GSH stores were kept unchanged. In a similar study, in which rats were injected with kainic acid to induce seizure, the expressions of the antioxidants superoxide dismutase and catalase were reduced in the hippocampus, while the antioxidants were elevated in the rats subjected to a treadmill exercise for 4 weeks, showing the preconditioning effect of exercise in suppressing oxidative stress by increasing hippocampal antioxidant enzymes [54]. It appears that this preconditioning effect of exercise becomes vital in maintaining oxidant-antioxidant balance upon an inflammatory challenge, as it is observed in seizure-induced neurotoxicity. On the other hand, exercise preconditioning was also shown to reduce neuroinflammation in a traumatic brain injury model by suppressing neutrophil infiltration [55]. When taken together with the associated reports, our data implicate that the neuronal oxidative damage produced by a single generalized seizure could be totally suppressed by exercise, suggesting that regular exercising in epileptic patients would have benefit in maintaining the oxidant-antioxidant status against the cumulative neurotoxicity due to repetitive epileptic seizures.

Depending on the training load, physical exercise can have positive effects on muscle function, while overproduction of ROS by a strenuous exercise can cause oxidative muscle damage [56]. In the current study, mild treadmill exercise for 10 days has enhanced generation of ROS with a concomitant increase in the endogenous antioxidant GSH levels, without causing any change in lipid peroxidation or neutrophil recruitment to the exercising muscle. Thus, it seems likely that the 10-day training has triggered ROS production and antioxidant capacity, which is expected to protect the exercised muscle from any upcoming oxidative challenges. Depending on the intensity of exercise, studies have reported that the endogenous antioxidants could be decreased or increased without any alteration in lipid peroxidation levels [57]. Although, high intensity exercise was shown to elevate myeloperoxidase activity in the gastrocnemius muscle $[58,59]$, the mild exercise in our study has not significantly affected the infiltration of neutrophils to the muscle, suggesting that a mild exercise is effective in upregulating the antioxidant content of the muscle without initiating any inflammatory response. On the other hand, exaggerated contraction of the muscle due to a PTZ-induced seizure in the current study has increased ROS generation, which was accompanied by the accumulation of neutrophils, while the GSH upregulated by exercise was used up to prevent any change in lipid peroxidation and to depress neutrophil infiltration. Thus, preconditioning of the muscle by prior exercise has increased the availability of the antioxidants to resist the oxidative injury evoked by generalized muscle contractions. In support of these biochemical data, our histological findings also revealed that abnormal organization of myofibrils and inflammatory cell infiltration in the muscles of the non-exercised PTZ rats were replaced by more regular myofibrils and a milder inflammation when the PTZ-rats have 
previously exercised. Due to the forces generated in the muscles during tonic-clonic seizures, epileptic patients have a high risk for severe musculoskeletal injury [60] and even rhabdomyolysis resulting in acute renal failure [61]. Although it is well known that patients experience severe pain following violent tonicclonic contractions, oxidative injury of the muscle induced by the seizure was not thoroughly investigated before. Therefore, our results verified for the first time that a single seizure enhances ROS generation and neutrophil accumulation, and initiates a local inflammatory response, which could give rise to soreness and pain experienced in the contracted muscles. Moreover, our results further showed that exercised and thereby preconditioned muscle could resist to the seizure-induced inflammatory insult.

In conclusion, the findings of the present study demonstrated that a mild exercise helps to maintain the oxidant-antioxidant balance in both the skeletal muscle and brain tissues against any forthcoming injurious incidents, and this preconditioning effect of exercise could be of vital importance in protecting the muscle and brain functions of the epileptic patients exposed to repetitive tonic-clonic contractions.

Acknowledgements: The authors would like to thank to Hilal Nişva Levent for her support in the preparation of histological samples.

\section{Compliance with Ethical Standards}

Ethical Approval: This study was approved by Marmara University Animal Care and Use Committee (approval code: 77.2017.mar).

Financial Support: No specific funding was received.

Conflict of Interest: There are no conflicting interests.

Author Contributions: SAT: Data acquisition (animal work, biochemical analyses). OTCK: Data acquisition (histological preparation and analyses). MY: Data acquisition (chemiluminescence analyses). AY: Design of work, data interpretation, critical revision of the manuscript. BCY: Design of work, data interpretation, critical revision of the manuscript. All authors contributed to the drafting of the manuscript and approved the final version of the article.

\section{REFERENCES}

[1] Horváth L, Fekete I, Molnár M, Válóczy R, Márton S, Fekete $\mathrm{K}$. The outcome of status epilepticus and long-term follow-up. Front Neurol 2019; 10:427-454. doi: 10.3389/fneur.2019.00427.

[2] Zhen J, Qu Z, Fang $H$, et al. Effects of grape seed proanthocyanidin extract on pentylenetetrazole-induced kindling and associated cognitive impairment in rats. Int J Mol Med 2014; 34:391-8. doi: 10.3892/ijmm.2014.1796.

[3] Mahmoudi T, Lorigooini Z, Rafieian-Kopaei M, et al. Effect of Curcuma zedoaria hydro-alcoholic extract on learning, memory deficits and oxidative damage of brain tissue following seizures induced by pentylenetetrazole in rat. Behav Brain Funct 2020; 16:1-12. doi: 10.1186/s12993.020.00169-3.
[4] Nassiri-Asl M, Mortazavi S-R, Samiee-Rad F, et al. The effects of rutin on the development of pentylenetetrazole kindling and memory retrieval in rats. Epilepsy Behav 2010; 18:50-3. doi: 10.1016/j.yebeh.2010.03.005.

[5] Power KN, Gramstad A, Gilhus NE, Hufthammer KO, Engelsen BA. Cognitive function after status epilepticus versus after multiple generalized tonic-clonic seizures. Epilepsy Res 2018; 140:39-45. doi: 10.1016/j.eplepsyres.2017.11.014.

[6] Shulman MB, Barr W. Treatment of memory disorders in epilepsy. Epilepsy Behav 2002; 3:30-4. doi: 10.1016/S15255050(02)00509-7.

[7] Sutter R, Dittrich T, Semmlack S, Rüegg S, Marsch S, Kaplan PW. Acute systemic complications of convulsive status Epilepticus-A systematic review. Crit Care Med 2018; 46:138-45. doi: 10.1097/CCM.000.000.0000002843.

[8] Liguori R, Giannoccaro MP, Pasini E, et al. Acute rhabdomyolysis induced by tonic-clonic epileptic seizures in a patient with glucose-6-phosphate dehydrogenase deficiency. J Neurol 2013; 260:2669-71. doi: 10.1007/s00415.013.7103-z.

[9] Malik GH. Rhabdomyolysis and myoglobin-induced acute renal failure. Saudi J Kidney Dis Transpl 1998; 9:273.

[10] Nguyen R, Zenteno JFT. Injuries in epilepsy: a review of its prevalence, risk factors, type of injuries and prevention. Neurol Int 2009; 1:72-78. doi: 10.4081/ni.2009.e20.

[11] Pandey S, Borah NC, Dwivedi M, Das RJ. Rhabdomyolysis with acute kidney injury after single episode of generalized seizure in a known case of epilepsy: a case report. Int J Res Med Sci 2020; 8:3757-60.

[12] Arida RM. Physical exercise and seizure activity. Biochim Biophys Acta Mol Basis Dis 2020:165979. doi: 10.1016/j. bbadis.2020.165979.

[13] Arida RM, de Almeida A-CG, Cavalheiro EA, Scorza FA. Experimental and clinical findings from physical exercise as complementary therapy for epilepsy. Epilepsy Behav 2013; 26:273-8. doi: 10.1016/j.yebeh.2012.07.025.

[14] van den Bongard F, Hamer HM, Sassen R, Reinsberger C. Sport and physical activity in epilepsy: A systematic review. Dtsch Arztebl Int 2020; 117:1. doi: 10.3238/arztebl.2020.0001.

[15] Gomes FN, Da Silva SG, Cavalheiro E, Arida R. Beneficial influence of physical exercise following status epilepticus in the immature brain of rats. Neuroscience 2014; 274:69-81. doi: 10.1016/j.neuroscience.2014.05.024.

[16] Sartori CR, Pelágio FC, Teixeira SA, et al. Effects of voluntary running on spatial memory and mature brain-derived neurotrophic factor expression in mice hippocampus after status epilepticus. Behav Brain Res 2009; 203:165-72. doi: 10.1016/j.bbr.2009.04.022.

[17] Campos DV, Lopim GM, de Almeida VS, Amado D, Arida RM. Effects of different physical exercise programs on susceptibility to pilocarpine-induced seizures in female rats. Epilepsy Behav 2016; 64:262-7. doi: 10.1016/j.yebeh.2016.08.011.

[18] Setkowicz Z, Mazur A. Physical training decreases susceptibility to subsequent pilocarpine-induced seizures in the rat. Epilepsy Res 2006; 71:142-8. doi: 10.1016/j. eplepsyres.2006.06.002. 
[19] Ding YH, Young CN, Luan X, et al. Exercise preconditioning ameliorates inflammatory injury in ischemic rats during reperfusion. Acta Neuropathol 2005; 109:237-46. doi: 10.1007/ s00401.004.0943-y.

[20] Li F, Geng X, Huber C, Stone C, Ding Y. In search of a dose: The functional and molecular effects of exercise on poststroke rehabilitation in rats. Front Cell Neurosci 2020; 14:186. doi: 10.3389/fncel.2020.00186.

[21] Powers SK, Bomkamp M, Ozdemir M, Hyatt H. Mechanisms of exercise-induced preconditioning in skeletal muscles. Redox Biology 2020; 35:101462. doi: 10.1016/j.redox.2020.101462.

[22] de Lima C, Arida RM, Andersen ML, et al. Effects of acute physical exercise in the light phase of sleep in rats with temporal lobe epilepsy. Epilepsy Res 2017; 136:54-61. doi: 10.1016/j.eplepsyres.2017.07.012.

[23] Koyuncuoğlu T, Arabacı Tamer S, Erzik C, et al. Oestrogen receptor ERa and ER $\beta$ agonists ameliorate oxidative brain injury and improve memory dysfunction in rats with an epileptic seizure. Exp Physiol 2019; 104:1911-28. doi: 10.1113/ EP087986.

[24] Racine R, Okujava V, Chipashvili S. Modification of seizure activity by electrical stimulation: III. Mechanisms. Electroencephalogr Clin Neurophysiol 1972; 32:295-9. doi: 10.1016/0013-4694(72)90178-2.

[25] Erşahin M, Toklu HZ, Erzik C, et al. The anti-inflammatory and neuroprotective effects of ghrelin in subarachnoid hemorrhage-induced oxidative brain damage in rats. J Neurotrauma 2010; 27:1143-55. doi: 10.1089/neu.2009.1210.

[26] Elrod K, Buccafusco JJ. An evaluation of the mechanism of scopolamine-induced impairment in two passive avoidance protocols. Pharmacol Biochem Behav 1988; 29:15-21. doi: 10.1016/0091-3057(88)90267-5.

[27] Bradley PP, Priebat DA, Christensen RD, Rothstein G. Measurement of cutaneous inflammation: estimation of neutrophil content with an enzyme marker. J Invest Dermatol 1982; 78:206-9. doi: 10.1111/1523-1747.ep12506462.

[28] Tuğtepe H, Şener G, Bıyıklı NK, et al. The protective effect of oxytocin on renal ischemia/reperfusion injury in rats. Regul Pept 2007; 140:101-8. doi: 10.1016/j.regpep.2006.11.026.

[29] Casini A, Ferrali M, Pompella A, Maellaro E, Comporti M. Lipid peroxidation and cellular damage in extrahepatic tissues of bromobenzene-intoxicated mice. Am J Pathol 1986; 123:520.

[30] Aykaç G, Uysal M, Yalçin AS, Koçak-Toker N, Sivas A, Öz H. The effect of chronic ethanol ingestion on hepatic lipid peroxide, glutathione, glutathione peroxidase and glutathione transferase in rats. Toxicology 1985; 36:71-6. doi: 10.1016/0300-483x(85)90008-3.

[31] Yildirim A, Tamer SA, Sahin D, et al. The effects of antibiotics and melatonin on hepato-intestinal inflammation and gut microbial dysbiosis induced by a short-term high-fat diet consumption in rats. Br J Nutr 2019; 122:841-55. doi: 10.1017/ S000.711.4519001466.

[32] Haklar G, Ulukaya-Durakbaś Ç, Yüksel M, Dagh T, Yalcin A. Oxygen radicals and nitric oxide in rat mesenteric ischaemia-reperfusion: Modulation by L-arginine and NG囚 nitro-L-arginine methyl ester. Clin Exp Pharmacol Physiol 1998; 25:908-12. doi: 10.1111/j.1440-1681.1998.tb02342.x.

[33] Ahmad N, Welch I, Grange R, et al. Use of imaging biomarkers to assess perfusion and glucose metabolism in the skeletal muscle of dystrophic mice. BMC Musculoskelet Disord 2011; 12:127. doi: 10.1186/1471-2474-12-127.

[34] Charge SB, Rudnicki MA. Cellular and molecular regulation of muscle regeneration. Physiol Rev 2004; 84:209-38. doi: 10.1152/physrev.00019.2003.

[35] Hawke TJ, Garry DJ. Myogenic satellite cells: physiology to molecular biology. J Appl Physiol 2001. doi: 10.1152/ jappl.2001.91.2.534.

[36] Auer R, Kalimo H, Olsson Y, Siesjö B. The temporal evolution of hypoglycemic brain damage. II. Light-and electronmicroscopic findings in the hippocampal gyrus and subiculum of the rat. Acta Neuropathol 1985; 67:25-36. doi: 10.1007/ BF00688121.

[37] Csordas A, Mazlo M, Gallyas F. Recovery versus death of" dark"(compacted) neurons in non-impaired parenchymal environment: light and electron microscopic observations. Acta Neuropathol 2003; 106:37-49. doi: 10.1007/ s00401.003.0694-1.

[38] Vasilev DS, Tumanova NL, Kim KK, et al. Transient morphological alterations in the hippocampus after pentylenetetrazole-induced seizures in rats. Neurochem Res 2018; 43:1671-82. doi: 10.1007/s11064.018.2583-y.

[39] Volk T. Positioning nuclei within the cytoplasm of striated muscle fiber: cooperation between microtubules and KASH proteins. Nucleus (Calcutta) 2013; 4:18-22. doi: 10.4161/ nucl.23086.

[40] Kaminski RM, Rogawski MA, Klitgaard H. The potential of antiseizure drugs and agents that act on novel molecular targets as antiepileptogenic treatments. Neurotherapeutics 2014; 11:385-400. doi: 10.1007/s13311.014.0266-1.

[41] Pimentel J, Tojal R, Morgado J. Epilepsy and physical exercise. Seizure 2015; 25:87-94. doi: 10.1016/j.seizure.2014.09.015.

[42] Kayacan Y, Ghojebeigloo BE, Çerit G, Kocacan SE, Ayyıldız M. Physical exercise and 5-hydroxytryptophan, a precursor for serotonin synthesis, reduce penicillin-induced epileptiform activity. Epilepsy Behav 2020; 112:107403. doi: 10.1016/j. yebeh.2020.107403.

[43] Arida RM, de Jesus Vieira A, Cavalheiro EA. Effect of physical exercise on kindling development. Epilepsy Res 1998; 30:12732. doi: 10.1016/s0920-1211(97)00102-2.

[44] McAuley JW, Long L, Heise J, et al. A prospective evaluation of the effects of a 12-week outpatient exercise program on clinical and behavioral outcomes in patients with epilepsy. Epilepsy Behav 2001; 2:592-600. doi: 10.1006/ebeh.2001.0271.

[45] Arida RM, Scorza FA, Scorza CA, Cavalheiro EA. Is physical activity beneficial for recovery in temporal lobe epilepsy? Evidences from animal studies. Neurosci Biobehav Rev 2009; 33:422-31. doi: 10.1016/j.neubiorev.2008.11.002.

[46] de Lima C, de Lira CAB, Arida RM, et al. Association between leisure time, physical activity, and mood disorder levels in 
individuals with epilepsy. Epilepsy Behav 2013; 28:47-51. doi: 10.1016/j.yebeh.2013.03.016.

[47] Chen L, Gong S, Shan L-D, et al. Effects of exercise on neurogenesis in the dentate gyrus and ability of learning and memory after hippocampus lesion in adult rats. Neurosci Bull 2006; 22:1-6.

[48] Devi PU, Manocha A, Vohora D. Seizures, antiepileptics, antioxidants and oxidative stress: an insight for researchers. Expert Opin Pharmacother 2008; 9:3169-77. doi: 10.1517/146.565.60802568230.

[49] Kudin AP, Kudina TA, Seyfried J, et al. Seizure冈dependent modulation of mitochondrial oxidative phosphorylation in rat hippocampus. Eur J Neurosci 2002; 15:1105-14. doi: 10.1046/j.1460-9568.2002.01947.x.

[50] Liu J, Wang A, Li L, Huang Y, Xue P, Hao A. Oxidative stress mediates hippocampal neuron death in rats after lithiumpilocarpine-induced status epilepticus. Seizure 2010; 19:16572. doi: 10.1016/j.seizure.2010.01.010.

[51] Sudha K, Rao AV, Rao A. Oxidative stress and antioxidants in epilepsy. Clin Chim Acta 2001; 303:19-24. doi: 10.1016/s00098981(00)00337-5.

[52] Golechha M, Sarangal V, Bhatia J, Chaudhry U, Saluja D, Arya DS. Naringin ameliorates pentylenetetrazol-induced seizures and associated oxidative stress, inflammation, and cognitive impairment in rats: possible mechanisms of neuroprotection. Epilepsy Behav 2014; 41:98-102. doi: 10.1016/j.yebeh.2014.09.058.

[53] Koyuncuoğlu T, Vızdıklar C, Üren D, et al. Obestatin improves oxidative brain damage and memory dysfunction in rats induced with an epileptic seizure. Peptides 2017; 90:37-47. doi: 10.1016/j.peptides.2017.02.005.

[54] Lee S-J. Effects of preconditioning exercise on nitric oxide and antioxidants in hippocampus of epileptic seizure. Journal of Exercise Rehabilitation 2019; 15:757. doi: 10.12965/ jer.1938698.349.

[55] Mota BC, Pereira L, Souza MA, et al. Exercise preconditioning reduces brain inflammation and protects against toxicity induced by traumatic brain injury: behavioral and neurochemical approach. Neurotox Res 2012; 21:175-84. doi: 10.1007/s12640.011.9257-8.

[56] Finaud J, Lac G, Filaire E. Oxidative stress. Sports Med 2006; 36:327-58. doi: 10.2165/00007.256.200636040-00004.

[57] Liu J, Yeo HC, Overvik-Douki E, et al. Chronically and acutely exercised rats: biomarkers of oxidative stress and endogenous antioxidants. J Appl Physiol 2000; 89:21-8. doi: 10.1152/ jappl.2000.89.1.21.

[58] Aoi W, Naito Y, Takanami Y, et al. Oxidative stress and delayedonset muscle damage after exercise. Free Radic Biol Med 2004; 37:480-7. doi:10.1016/j.freeradbiomed.2004.05.008.

[59] Morozov VI, Tsyplenkov PV, Golberg ND, Kalinski MI. The effects of high-intensity exercise on skeletal muscle neutrophil myeloperoxidase in untrained and trained rats. Eur J Appl Physiol 2006; 97:716-22. doi: 10.1007/s00421.006.0193-x.

[60] Mehlhorn A, Strohm P, Hausschildt O, Schmal H, Sudkamp N. Seizure-induced muscle force can caused lumbar spine fracture. Acta Chir Orthop Traumatol Cech 2007; 74:202.

[61] Mishra A, Dave N. Acute renal failure due to rhabdomyolysis following a seizure. J Family Med Prim Care 2013; 2:86-7. doi: 10.4103/2249-4863.109962. 